\title{
BMJ Open Impact of preoperative HbA1c on postoperative complications after elective major abdominal surgery: a systematic review protocol
}

\author{
Joanna Kae Ling Wong (D) , ${ }^{1}$ Yuhe $\mathrm{Ke},{ }^{2}$ Yi Jing Ong, ${ }^{3}$ Hui Hua Li, ${ }^{4}$ \\ Hairil Rizal Abdullah (D) 2,5
}

To cite: Wong JKL, Ke Y, Ong YJ, et al. Impact of preoperative $\mathrm{HbA} 1 \mathrm{c}$ on postoperative complications after elective major abdominal surgery: a systematic review protocol. BMJ Open 2020;10:e039422. doi:10.1136/ bmjopen-2020-039422

- Prepublication history for this paper is available online. To view these files, please visit the journal online (http://dx.doi org/10.1136/bmjopen-2020039422).

Received 15 April 2020 Revised 16 August 2020 Accepted 18 August 2020

\section{Check for updates}

(c) Author(s) (or their employer(s)) 2020. Re-use permitted under CC BY-NC. No commercial re-use. See rights and permissions. Published by BMJ.

${ }^{1}$ Department of Medicine, Northwick Park Hospital, London, UK

${ }^{2}$ Division of Anaesthesiology and Perioperative Medicine, Singapore General Hospital, Singapore

${ }^{3}$ Yong Loo Lin School of

Medicine, National University of

Singapore, Singapore

${ }^{4}$ Health Services Research Unit,

Singapore General Hospital,

Singapore

${ }^{5}$ DukeNUS Medical School,

Singapore

Correspondence to Dr Hairil Rizal Abdullah; hairil.rizal.abdullah@singhealth. com.sg

\section{ABSTRACT}

Introduction Diabetes has an increasing worldwide prevalence. It is known to be a predisposing factor for postoperative complications. Preoperative glycaemic control strategies should be pursued as glycaemic control could serve as a modifiable risk factor. Glycated haemoglobin (HbA1c), a marker of 3-month average glycaemic control, has been shown in meta-analyses to predict postoperative complications in cardiothoracic, bariatric and orthopaedic surgery. However, there is no meta-analysis in the major abdominal surgery population, in whom morbidity may be higher due to the nature of the surgery. Understanding the association between $\mathrm{HbA1C}$ and postoperative complications could help in preoperative risk prognostication, counselling and glycaemic target selection. The aim of this systematic review and meta-analysis is to evaluate all evidence on the association between preoperative $\mathrm{HbA} 1 \mathrm{C}$ and postoperative complications in elective major abdominal surgery, and to investigate the threshold $\mathrm{HbA} 1 \mathrm{c}$ level before postoperative complication rates increase.

Methods and analysis This review will be performed according to Preferred Reporting Items for Systematic Reviews and Meta-Analyses Protocols guidelines. PubMed, Embase, Cochrane Central Register of Controlled Trials, Google Scholar and China National Knowledge Infrastructure will be searched for all original studies. Study selection, data extraction, risk of bias and quality assessment will be conducted by two independent reviewers. The primary outcome is the association between preoperative $\mathrm{HbA} 1 \mathrm{c}$ and major postoperative complications (Clavien Dindo 3-5), and the secondary outcome is the association between $\mathrm{HbA} 1 \mathrm{c}$ and overall postoperative complications. Data management and synthesis will be performed using Microsoft Excel and Stata to derive pool estimates.

Ethics and dissemination No ethics approval is required as only secondary data will be used. Findings will be disseminated through peer-reviewed journals and conference presentations.

PROSPERO registration number CRD42020167347.

\section{INTRODUCTION}

\section{Background}

Diabetes mellitus is known to be a significant predisposing factor for postoperative complications such as infection, sepsis, poor wound
Strengths and limitations of this study

- This is the first meta-analysis investigating the association between preoperative glycated haemoglobin $(\mathrm{HbA} 1 \mathrm{c})$ and postoperative complications in the patient population undergoing elective major abdominal surgery.

- A total of six databases, including a Chinese database, will be searched to provide a comprehensive range of studies for inclusion.

- A meta-regression will be performed to evaluate the effect of different $\mathrm{HbA1c}$ cut-off values on postoperative outcomes.

- The varying outcome measures reported by individual studies may limit the performance of a quantitative analysis, in which case, a qualitative synthesis will be performed.

- The variety of postoperative complications reported by individual studies provides the opportunity to study postoperative complications as guided by the American College of Surgeons National Surgical Quality Improvement Programme.

healing, anastomotic leak, water-electrolyte and acid-base imbalances, cardiac complications, acute kidney injury and more. ${ }^{1}$ Furthermore, when compared with patients with non- diabetes, both the in-hospital mortality and long-term mortality are considerably higher in diabetic patients. ${ }^{2}$ Hence, strategies for glycaemic control in the perioperative period have received much attention as glycaemic control could serve as a modifiable risk factor and a potential target for reducing complications following surgery.

The American Diabetes Association endorses glycated haemoglobin (HbAlc) for monitoring glycaemic control among patients with diabetes. ${ }^{3}$ This is a measure reflecting the 3-month average blood glucose level of patients, hence giving an indirect measurement of how effectively an individual's blood glucose is controlled. ${ }^{4}$ It has been 
conclusively shown through published systematic reviews and meta-analyses that increased levels of preoperative HbAlc is strongly associated with higher rates of postoperative complications or poorer outcomes in surgical specialties such as cardiothoracic, ${ }^{56}$ bariatric $^{78}$ and orthopaedic surgery. ${ }^{9} 10$

Major abdominal surgery, defined as a major operation involving the abdominal and/or retroperitoneal compartment with the removal of all or part of an organ (stomach, liver, kidney, bowel, bladder) with a realistic chance of blood loss of more than $500 \mathrm{~mL}$ and surgery lasting at least 1 hour, is associated with high postoperative morbidity due to the extensive nature of the surgery. Despite the clinical significance of this, there has not been a systematic review or meta-analysis investigating the association between preoperative HbAlc and postoperative complications in this patient population, after a preliminary search of multiple databases including PROSPERO, MEDLINE (via Ovid), the Cochrane Database of Systematic Reviews and the Joanna Briggs Institute Database of Systematic Reviews and Implementation Reports. Furthermore, there is no consensus on the HbA1c threshold at which postponing elective surgery is warranted. Joint British Diabetes Societies for Inpatient Care and The Association of Anesthetists of Great Britain and Ireland recommend further optimisation of glycaemic control at a HbA1c threshold of $8.5 \%,{ }^{11}$ while the US Society for Ambulatory Anesthesia recommends a threshold of $7.0 \%{ }^{12}$ and the Australian Diabetes Society recommends a threshold of $9.0 \% .^{13}$

There is a gap in research as the association between preoperative $\mathrm{HbAlc}$ and postoperative complications in major abdominal surgery is unknown despite the increasing incidence of both diabetes and abdominal surgery. Diabetes is a major public health problem that has reached global pandemic status with a worldwide prevalence of $9.3 \%$ in 2019 , forecasted to rise to $10.2 \%$ by $2030 .{ }^{14}$ Similarly, the incidence of non-communicable diseases such as cancer ${ }^{15} 16$ requiring major abdominal surgery is increasing. Data from the UK National Diabetes Inpatient Audit in 2013 showed that prevalence of diabetes among inpatients in the UK range from $10 \%$ to $35 \%$, and at least $10 \%$ of all surgical patients have diabetes. ${ }^{17}$

Further insight into the association between preoperative $\mathrm{HbAlc}$ and postoperative complications after elective major abdominal surgery could potentially have a great impact on public health. The findings from this proposed systematic review and meta-analysis will help in risk prognostication and counselling prior to major abdominal surgery in patients with diabetes. Furthermore, knowledge obtained from this review may guide the selection of appropriate glycaemic targets to be achieved prior to elective major abdominal surgery.

\section{Objective}

The aim of this systematic review and meta-analysis is to evaluate all currently available evidence on the association between preoperative HbAlc and postoperative complications in patients undergoing elective major abdominal surgery. Furthermore, we will investigate if there is a threshold HbAlc level before the rate of postoperative complications increases.

\section{METHODS}

This protocol has been written according to the Preferred Reporting Items for Systematic Reviews and Meta-Analyses (PRISMA) Protocols 2015 checklist. ${ }^{18}$

\section{Eligibility criteria}

The eligibility criteria used to include and exclude studies are as follows:

\section{Study design}

Randomised controlled trials (RCTs) and observational studies will be included. Retrospective studies will be included if they constitute data collection from patient notes, and excluded if they consist of patient interviews or any data collection method subject to recall or responder bias. Abstracts, case reports, systematic reviews, metaanalyses, comments, letters to the editor and expert opinions will be excluded.

\section{Population}

Studies including adult patients undergoing elective major abdominal surgery (stomach, liver, kidney, bowel, bladder, oesophagus) who have a recorded $\mathrm{HbA1c}$ will be included. Studies on patients undergoing bariatric, total pancreatectomy, paediatric, emergency and transplant surgery will be excluded.

\section{Intervention and comparator}

We will include studies which use at least two HbA1c groups, where the group with the low HbAlc cut-off will be deemed the 'intervention group' and the group with the high HbA1c cut-off will be deemed the 'comparator group'. Studies that do not measure HbAlc or do not have at least two HbA1c groups for comparison purposes will be excluded.

\section{Outcome}

Studies that evaluate any postoperative complications (30 days and 90 days) as the outcome measure will be included. ${ }^{19}$ Studies that do not evaluate postoperative complications as their outcome measure will be excluded.

\section{Language}

Studies in the English and Chinese language will be included as they are the languages which we are confident can be translated and analysed accurately by the authors.

\section{Information sources}

Electronic searches will be performed in the following databases: PubMed, Embase, MEDLINE, Cochrane Central Register of Controlled Trials, Google Scholar, China National Knowledge Infrastructure (CNKI). A 
manual search of citations and references of eligible studies will also be conducted. All records up to the 1 April 2020 will be included.

\section{Search strategy}

The search strategy will be developed with a research librarian using the terms below:

( (hba1c) OR (glycated h(a) emoglobin a1c) OR (glyc (a) emic control) OR (glycosylated haemoglobin a1c)) AND ( (abdominal surgery) OR (general surgery) OR (lower gastrointestinal surgery) OR (colorectal surgery) OR (upper gastrointestinal surgery) OR (urology))

Records up to the 1 April 2020 will be included.

\section{Study records}

\section{Data management}

Records will be exported from the electronic databases to Microsoft Excel and all studies collated. Duplicates will be removed using the 'Remove duplicates' function and through manual searching if undetected by Microsoft Excel (due to differences in spelling or punctuation in the study title). The number of records will be noted before and after removing duplicates and at each step of the study selection process to produce an accurate PRISMA flow chart.

\section{Selection process}

Study selection will be performed by two independent reviewers (JKLW and YK). The first stage of selection will involve screening of the study titles and abstracts, and the categorisation of records as 'Yes', 'No' and 'Maybe'. The second stage of selection will involve full-text screening of studies labelled 'Yes' and 'Maybe', and categorisation of these studies into 'Yes', 'No' and 'Maybe'. All studies labelled 'Maybe' will be discussed between JKLW and YK. If no consensus is achieved, a third reviewer (HRA) will resolve them. At all times, selection will be performed based on the predetermined inclusion and exclusion criteria. If studies are excluded, the reason for exclusion will be noted.

\section{Data collection process}

Data collection from included studies will be performed by two independent reviewers (JKLW and YK) and stored on pre-made proformas on separate Microsoft Excel sheets. All efforts will be made to obtain data from the original study authors if not readily available in the main publication or online supplemental material.

\section{Data items}

These are the data items from studies that will be extracted:

- Data on study characteristics will include author, year, journal, city and country of study, study design and setting (eg, district hospital, tertiary hospital). We will determine the study's nationality based on the reported study population. For multinational, multicentre studies, we will assign the study nationality based on the corresponding author's institution.
- Population data will include patient demographics (age and gender), type of surgery, total sample size, number of patients in each $\mathrm{HbAlc}$ group and number of patients lost to follow-up if applicable.

- Intervention and comparator data will include the number of groups or dichotomies of HbAlc levels used, and the HbAlc threshold used for dichotomisation. If the HbA1c level is expressed in the International Federation of Clinical Chemistry unit (mmol/ $\mathrm{mol}$ ), we will use an online calculator to convert it to the Diabetes Control and Complications Trial unit $(\%) .{ }^{20}$

- Outcome data will include all postoperative complications including but not limited to serious complications, any complications, pneumonia, cardiac complication, surgical site infection, urinary tract infection, venous thromboembolism, renal failure, readmission, return to theatre, death and sepsis, following the American College of Surgeons National Surgical Quality Improvement Programme. ${ }^{21}$

- Whenever possible, the following information will be extracted: (1) the number of patients involved in the treatment and control groups; (2) the OR/hazards ratio (HR) with $95 \%$ CI for binary outcome/time-toevent outcome with number of events, mean together with SD or $95 \%$ CI for continuous outcome.

- Data on funding sources and conflict of interests will be extracted if available to the study.

\section{Outcomes and prioritisation}

The primary outcome of this systematic review and metaanalysis is the association between preoperative HbAlc and major postoperative complications fulfilling the Clavien Dindo (CD) classification grade $3-5,{ }^{19}$ following a widely accepted standard definition of 'major postoperative complications' as CD 3-5. ${ }^{22-25}$

- Grade 3: Complications requiring surgical, endoscopic or radiological intervention.

- Grade 4: Life-threatening complications requiring intensive care unit management.

- Grade 5: Death.

The secondary outcome of this systematic review and meta-analysis is the association between preoperative HbA1c and overall complications fulfilling CD grade $1-5 .^{19}$

- Grade 1: Any deviation from the normal postoperative course without the need for pharmacological treatment or surgical, endoscopic, and radiological interventions. Allowed therapeutic regimens are antiemetics, antipyretics, analgesia, diuretics, electrolytes and physiotherapy. This grade also includes wound infections opened at the bedside.

- Grade 2: Any deviation from the normal postoperative course requiring pharmacological treatment with drugs apart from those allowed in grade 1, blood transfusions and total parenteral nutrition.

- Grade 3-5: as above. 


\section{Risk of bias and quality assessment}

Risk of bias and quality assessments will be performed independently by two reviewers (YK and YJO). Any disagreements will be resolved with a third reviewer (HRA). The risk of bias for non-randomised observational studies included will be assessed using the NewcastleOttawa Quality Assessment Scale (NOS) across three quality parameters (study selection, comparability of the population and a determination of whether the exposure or outcome includes the risk of bias) and scored out of nine points. The scores from the NOS will be converted to The Agency for Healthcare Research and Quality standards, classifying studies into 'good quality', 'fair quality' and 'poor quality'. ${ }^{26}$

The risk of bias for RCTs will be assessed using the Cochrane risk of bias tool outlined by The Cochrane Handbook for Systematic Reviews of Interventions across seven domains: random sequence generation, allocation concealment, blinding of participants and personnel, blinding of outcome assessment, incomplete outcome data, selective outcome reporting and other biases. ${ }^{27}$

\section{Data synthesis and meta-bias}

The complications extracted from studies will initially be graded according to the CD classification. Complications will then be grouped for analysis into either primary and secondary outcome; primary if they are CD3 and above (major complications), and secondary if they are CD1 and above (overall complications). Secondary outcomes will be further categorised into any complications, pneumonia, cardiac complication, surgical site infection, urinary tract infection, venous thromboembolism, renal failure, readmission, return to theatre, death and sepsis. The number of postoperative complications for both the lower HbA1c and higher HbA1c group will be used to derive pool estimates for each postoperative complication. HbAlc will be treated as a binary variable as this is the format in which most primary studies report their complications.

Statistical analyses will be performed using Stata Statistical Software (StataCorp. 2019. Stata Statistical Software: Release V.16, StataCorp). Funnel plots, together with Begg's rank correlation test and Egger's regression asymmetry test will be used to assess publication bias. ${ }^{28}$ In addition, the Duval and Tweedie non-parametric 'trim and fill' method of accounting for publication bias will be performed to formalise the use of funnel plots and adjust the meta-analysis by incorporating the theoretical missing trials. ${ }^{28}$ Q-statistic will be used to investigate the degree of heterogeneity between studies. As a limitation of Cochran's Q-test is the fact that it might be underpowered when few studies have been included or when event rates are low, standard practice is to adopt a higher $p$ value (rather than 0.05) as threshold for statistical significance. Thus, a $\mathrm{p}>0.1$ will be interpreted as evidence by chance alone. $\mathrm{I}^{2}$ statistical test ${ }^{29}$ will be carried out to describe the proportion of total variation caused by heterogeneity because the Q-statistic has low power in common situations of few studies and excessive power to detect clinically unimportant heterogeneity when there are many studies. ${ }^{30} \mathrm{I}^{2}$ of less than $30 \%$ of the variability in point estimate will be considered as mild heterogeneity, more than $50 \%$ as notable heterogeneity, whereas anything in between considered as moderate heterogeneity. The random effects model (DerSimonian-Laird method) will be used to derive pool estimates and generate forest plots to account for interstudy heterogeneity, to see the effect of low and elevated HbAlc on the various postoperative complications. A meta-regression will be performed to evaluate the effect of different HbA1c cut-off values on the outcome. If a meta-analysis is not possible, a qualitative synthesis will be performed instead.

\section{Patient and public involvement}

Patients or the public will not be involved in the design, conduct, reporting or dissemination plans at this stage of our research. Patient and public involvement will be necessary in future implementation studies.

\section{Role of study team members}

HRA and JKLW initiated the study. HRA, JKLW, YK, YJO and HHL participated in study design. JKLW, YK, YJO and HHL will write the full study manuscript with critical appraisal and further development by HRA and an additional author to represent a surgical perspective. HHL will advise regarding statistical and data analyses.

\section{DISCUSSION}

Conducting this meta-analysis will answer the research question of whether preoperative HbAlc has an association with postoperative complications in patients undergoing elective major abdominal surgery, a population which has not yet been investigated through a meta-analysis.

The rationale for excluding certain patient populations will be explained here. Patients undergoing pancreatic surgery will be excluded from the definition of 'Major abdominal surgery' because the postoperative glucose metabolism in these patients are different to those undergoing other types of abdominal surgery. ${ }^{31}$ As perioperative glucose control has been demonstrated to be an independent predictor of postoperative complications, ${ }^{32} 33$ it would be inappropriate to group pancreatic surgery patients with other types of surgeries with an intact pancreas. Bariatric patients will be excluded as these patients have altered postoperative glucose metabolism too. The mechanism in which this is different is associated with hormones such as incretin and glucagon-like peptide $1 .{ }^{34}{ }^{35}$ Patients undergoing emergency surgery will be excluded as these patients are fundamentally different to those undergoing elective surgery, as they are by default already subject to higher postoperative complications due to the nature of the surgery (eg, the unprepared bowel, faecal contamination, haemodynamic instability, sepsis). Additionally, for the future intent of 
our study which is to optimise preoperative HbAlc in the preoperative setting, preoperative HbA1c optimisation would be irrelevant for patients undergoing emergency surgery as they would not have the luxury of the 'preoperative period'. Finally, transplant patients will be excluded as the nature of transplant surgery is unique to that of 'major abdominal surgery', as defined in the introduction.

A strength of this meta-analysis is the fact that it will give insight into the association between preoperative HbA1c and postoperative complications in a population not investigated before through a meta-analysis, that is, the major abdominal surgery population. Furthermore, the inclusion of the Chinese database, CNKI, will ensure extensive coverage of literature as the database has grown significantly in the past decade.

The limitations in study design will be discussed here. While the wide variety of postoperative complications investigated by individual studies is an advantage as it allows insight to be gained on the association between preoperative HbAlc and various types of postoperative complications, this leads to the inevitability that there will be some postoperative complications that are only investigated by one or two studies, limiting the potential for a meta-analysis for these complications. In these cases, a qualitative analysis will be done. Another potential limitation is the fact that HbAlc levels will be categorised into two groups (binary variable), that is, 'Elevated' and 'Low' HbAlc to derive pool estimates, while in reality HbAlc is a continuous variable. However, a preliminary search found that primary studies report their complications by similarly treating HbAlc as a binary variable. Therefore, treating HbAlc as a binary variable would be the optimal way to respect the original data from the primary studies.

\section{Acknowledgements The authors would like to thank the Health Services Research Unit of Singapore General Hospital for their assistance and support in conducting this review.}

Contributors HRA and JKLW initiated the study. HRA, JKLW, YK and YJO participated in study design. JKLW, YK and YJO drafted the manuscript with critical appraisal and further development by HRA. HHL advised regarding statistical and data analyses. All authors contributed to the revision of the manuscript and approval of the final version. HRA is the guarantor of the review.

Funding This work was supported by the department fund of the Department of Anaesthesiology, Singapore General Hospital, Singapore. HRA is a recipient of SingHealth Duke-NUS Nurturing Clinician Scientists Scheme Award, project number 12/FY2017/P1/15-A29.

Disclaimer The funding sources have no role in the design of this study and the analysis and interpretation of the results.

\section{Competing interests None declared.}

Patient and public involvement Patients and/or the public were not involved in the design, or conduct, or reporting, or dissemination plans of this research.

\section{Patient consent for publication Not required}

Provenance and peer review Not commissioned; externally peer reviewed.

Open access This is an open access article distributed in accordance with the Creative Commons Attribution Non Commercial (CC BY-NC 4.0) license, which permits others to distribute, remix, adapt, build upon this work non-commercially, and license their derivative works on different terms, provided the original work is properly cited, appropriate credit is given, any changes made indicated, and the use is non-commercial. See: http://creativecommons.org/licenses/by-nc/4.0/.
ORCID iDs

Joanna Kae Ling Wong http://orcid.org/0000-0001-5498-2212

Hairil Rizal Abdullah http://orcid.org/0000-0003-1916-0832

\section{REFERENCES}

1 Wang J, Chen K, Li X, et al. Postoperative adverse events in patients with diabetes undergoing orthopedic and general surgery. Medicine 2019;98:e15089.

2 Krolikowska M, Kataja M, Pöyhiä R, et al. Mortality in diabetic patients undergoing non-cardiac surgery: a 7-year follow-up study. Acta Anaesthesiol Scand 2009;53:749-58.

3 American Diabetes Association. Standards of medical care in diabetes-2011. Diabetes Care 2011;34:S11-61.

4 Sacks DB. Correlation between hemoglobin A1c $(\mathrm{HbA1c})$ and average blood glucose: can $\mathrm{HbA1c}$ be reported as estimated blood glucose concentration? J Diabetes Sci Technol 2007;1:801-3.

5 Zheng J, Cheng J, Wang T, et al. Does HbA1c level have clinical implications in diabetic patients undergoing coronary artery bypass grafting? A systematic review and meta-analysis. Int J Endocrinol 2017;2017:1537213

6 Biancari F, Giordano S. Glycated hemoglobin and the risk of sternal wound infection after adult cardiac surgery: a systematic review and meta-analysis. Semin Thorac Cardiovasc Surg 2019;31:465-7.

7 Chow A, Switzer NJ, Dang J, et al. A systematic review and metaanalysis of outcomes for type 1 diabetes after bariatric surgery. $J$ Obes 2016;2016:6170719

8 Wang G-F, Yan Y-X, Xu N, et al. Predictive factors of type 2 diabetes mellitus remission following bariatric surgery: a meta-analysis. Obes Surg 2015;25:199-208.

9 Yang L, Sun Y, Li G, et al. Is hemoglobin A1c and perioperative hyperglycemia predictive of periprosthetic joint infection following total joint arthroplasty?: a systematic review and meta-analysis. Medicine 2017;96:e8805.

10 Shohat N, Muhsen K, Gilat R, et al. Inadequate glycemic control is associated with increased surgical site infection in total joint arthroplasty: a systematic review and meta-analysis. J Arthroplasty 2018;33:2312-21.

11 Dhatariya K, Levy N, Kilvert A, et al. NHS diabetes guideline for the perioperative management of the adult patient with diabetes. Diabet Med 2012;29:420-33.

12 Joshi GP, Chung F, Vann MA, et al. Society for ambulatory anesthesia consensus statement on perioperative blood glucose management in diabetic patients undergoing ambulatory surgery. Anesth Analg 2010;111:1378-87.

13 Australian Diabetes Society. Peri-operative diabetes management guidelines. Aust Diabetes Soc 2012;30.

14 Unnikrishnan R, Pradeepa R, Joshi SR, et al. Type 2 diabetes: demystifying the global epidemic. Diabetes 2017;66:1432-42.

15 Vuik FE, Nieuwenburg SA, Bardou M, et al. Increasing incidence of colorectal cancer in young adults in Europe over the last 25 years. Gut 2019;68:1820-6.

16 Rawla P, Sunkara T, Muralidharan P, et al. Update in global trends and aetiology of hepatocellular carcinoma. Contemp Oncol 2018;22:141-50.

17 Centre H and SCI. National diabetes inpatient audit (NaDIA), 2014. Available: http://www.hscic.gov.uk/catalogue/PUB14358

18 Moher D, Shamseer L, Clarke M, et al. Preferred reporting items for systematic review and meta-analysis protocols (PRISMA-P) 2015 statement. Syst Rev 2015;4:1.

19 Dindo D, Demartines N, Clavien P-A. Classification of surgical complications: a new proposal with evaluation in a cohort of 6336 patients and results of a survey. Ann Surg 2004;240:205-13.

20 Diabetes. Hba1C units converter - DCCT to IFCC, 2019. Available: https://www.diabetes.co.uk/hba1c-units-converter.html [Accessed 14 Mar 2020].

21 Surgeons TAC. ACS national surgical quality improvement program, 2020.

22 McMahon JD, Maclver C, Smith M, et al. Postoperative complications after major head and neck surgery with free flap repair--prevalence, patterns, and determinants: a prospective cohort study. Br J Oral Maxillofac Surg 2013;51:689-95.

23 Rassweiler JJ, Rassweiler M-C, Michel M-S. Classification of complications: is the Clavien-Dindo classification the gold standard? Eur Urol 2012;62:256-8.

24 Umezawa $\mathrm{H}$, Nakao J, Matsutani T, et al. Usefulness of the claviendindo classification in understanding the limitations and indications of Larynx-preserving esophageal reconstruction. Plast Reconstr Surg Glob Open 2016;4:e1113. 
25 European Association Urology. European association of urology guidelines. 2020 edn. Arnhem, The Netherlands: European Association of Urology Guidelines Office, 2020.

26 Wells G, Sheah B, O'Connell D, et al. The Newcastle-Ottawa scale (NOS) for assessing the quality of nonrandomised studies in metaanalyses, 2000. Available: http://www.ohri.ca/programs/clinical_ epidemiology/oxford.asp [Accessed 14 Mar 2020].

27 Higgins J, Thomas J, Chandler J, et al. Cochrane handbook for systematic reviews of interventions version 6.0 (updated July 2019), 2019. Available: www.training.cochrane.org/handbook

28 Sutton AJ, Abrams KR, Jones DR, et al. Methods for meta-analysis in medical research. Chichester: John Wiley \& Sons Ltd, 2000.

29 Higgins JPT, Thompson SG. Quantifying heterogeneity in a metaanalysis. Stat Med 2002;21:1539-58.

30 Hardy RJ, Thompson SG. Detecting and describing heterogeneity in meta-analysis. Stat Med 1998;17:841-56.
31 Wu J-M, Ho T-W, Yang C-Y, et al. Changes in glucose metabolism after distal pancreatectomy: a nationwide database study. Oncotarget 2018;9:11100-8.

32 Kwon S, Thompson R, Dellinger P, et al. Importance of perioperative glycemic control in general surgery: a report from the surgical care and outcomes assessment program. Ann Surg 2013;257:8-14.

33 Pomposelli JJ, Baxter JK, Babineau TJ, et al. Early postoperative glucose control predicts nosocomial infection rate in diabetic patients. JPEN J Parenter Enteral Nutr 1998;22:77-81.

34 Smith EP, Polanco G, Yaqub A, et al. Altered glucose metabolism after bariatric surgery: what's GLP-1 got to do with it? Metabolism 2018;83:159-66.

35 Holter MM, Dutia R, Stano SM, et al. Glucose metabolism after gastric banding and gastric bypass in individuals with type 2 diabetes: weight loss effect. Diabetes Care 2017;40:7-15. 